On the Linearization of Nonlinear Control Systems and Exact Reachability

\title{
Halina Frankowska*
}

September 1987

WP-87-086

*CEREMADE, Université Paris-Dauphine, 75775 Paris Cx 16, France

INTERNATIONAL INSTITUTE FOR APPLIED SYSTEMS ANALYSIS

A-2361 Laxenburg, Austria 


\section{Foreword}

The author studies the problem of exact local reachability of infinite dimensional

nonlinear control systems. The main result shows that the exact local reachability of a linearized system implies that of the original system. The main tool is an inverse mapping theorem for a map from a complete metric space to a reflexive Banach space.

Alexander B. Kurzhanski

Chairman

Systems and Decision Sciences Program 


\title{
On the Linearization of Nonlinear Control Systems and Exact Reachability
}

\author{
Halina Frankowska *
}

\section{Introduction.}

Consider the following abstract control system

$$
\left\{\begin{array}{l}
x^{\prime}=A x+f(x, u), u \in U \\
x(0)=x_{0}
\end{array}\right.
$$

where $A$ is the infinitesimal generator of a strongly continuous semigroup of linear operators. Let $R(T)$ denote the reachable set of (1.1) at time $T$ (by the mild solutions of (1.1)). Consider a mild solution $\bar{x}$ and let $\bar{u}$ be the corresponding control.

We address here the following two questions:

1. Does $\bar{x}(T) \in \operatorname{Int} R(T)$ ?

2. Given a point $y$ near $\bar{x}(T)$ how much we have to change the control $\bar{u}$ in order the trajectory $x$ corresponding to this new control satisfies $x(T)=y$ ?

We study the above via a linearization technique and an inverse mapping theorem for a map whose domain of definition is a complete metric space.

Let us explain now what do we mean by linearization. In control theory linearization is usually understood as a substitution of the nonlinear system (1.1) by the linear system

$$
\left\{\begin{array}{l}
w^{\prime}(t)=A w(t)+\frac{\partial f}{\partial x}(\bar{x}(t), \bar{u}(t)) w(t)+\frac{\partial f}{\partial u}(\bar{x}(t), \bar{u}(t)) u \\
w(0)=0
\end{array}\right.
$$

where the controls $u$ belong to the space spanned by $U$. To make use of such linearization it is usually required that $\bar{u}(t) \in \operatorname{Int} U$ or even that $U$ is a Banach space (see for example [19]). 
Studying a particular trajectory $\bar{x}$ we can not guarantee such property (unless the set $U$ is open for example equal to a Banach space). Another linearization of (1.1) along $(\bar{x}, \bar{u})$ is given by the following linear control system

$$
\left\{\begin{array}{l}
w^{\prime}(t)=A w(t)+\frac{\partial f}{\partial x}(\bar{x}(t), \bar{u}(t)) w(t)+v(t) \\
v(t) \in \bar{c} o f(\bar{x}(t), U)-f(\bar{x}(t), \bar{u}(t)) \\
w(0)=0
\end{array}\right.
$$

Here we do not have any restrictions on the control $\bar{u}$ and thus we can apply it to any trajectory-control pair $(\bar{x}, \bar{u})$.

The two linearizations (1.2) and (1.3) are related in the following way: If $\bar{u}(t) \in \operatorname{Int} U$ then for every $u, \frac{\partial}{\partial u} f(\bar{x}(t), \bar{u}(t)) u \in T_{c o f(\bar{x}(t), U)}\left(f(\bar{x}(t), \bar{u}(t))\right.$, where $T_{K}(x)$ denote the tangent cone (of convex analysis) to a convex set $K$ at $x \in K$.

The second linearization was used in [22] to get local reachability of a nonlinear finite dimensional control system via the local reachability of the linearized system. It seems that the result of [22] does not have yet its analogue in infinite dimensional spaces and we shall prove it here in Section 3. Namely we show that if zero is an interior point of the reachable set $R^{L}(T)$ of the linear system (1.3) at time $T$, then $\bar{x}(T) \in \operatorname{Int} R(T)$, i.e. we obtain a sufficient condition to answer positively the Question 1. We also show that $0 \in \operatorname{Int} R^{L}(T)$ implies the existence of $L>0$ such that for every $y$ near $\bar{x}(T)$, there exists a trajectory-control pair $(x, u)$ satisfying

$$
x(T)=y, \mu(\{t: u(t) \neq \bar{u}(t)\}) \leq L|x(T)-y|
$$

where $\mu$ denotes the Lebesgue measure. This second result seems to be unknown even in the finite dimensional case.

To prove the above, we need a very general inverse mapping theorem for maps whose domain of definition is a complete metric space. This will allow us to avoid difficult constructions of "fixed point argument" type proofs. Let us explain briefly how. We assume that $f$ is so that to every admissible control $u$ corresponds the unique mild solution $x_{u}$ of (1.1). Consider the map $U_{a d} \ni u \rightarrow{ }^{G} x_{u}(T)$.

In 1934 Ljusternik [13] proved that if a $C^{1}$-function $G: U \rightarrow X$ between two Banach spaces has a surjective derivative $G^{\prime}(\bar{u})$ at a point $\bar{u} \in U$, then for all $h>0, G(\bar{u}) \in$ Int $G\left(B_{h}(\bar{u})\right)$ (i.e., the open mapping principle holds true) and the setvalued map $G^{-1}$ is roughly speaking Lipschitzian at $\bar{u}$. 


\section{An Inverse Function Theorem}

Consider a complete metric space $(U, d)$, a reflexive Banach space $X$ and a continuous map $G: U \rightarrow X$ is Gâteaux differentiable away from zero. For all $u \in U, h>0$ let $B_{h}(u)$ denote the closed ball in $U$ of center $u$ and radious $h$. Let $\bar{u} \in U$ be a given point. In this section we study a sufficient condition for:

$$
\underset{h>0}{\forall} G(\bar{u}) \in \operatorname{Int} G\left(B_{h}(\bar{u})\right) \text { (open mapping principle) }
$$

and the regularity of the inverse map $G^{-1}: X \rightarrow U$ given by

$$
G^{-1}(x)=\{u \in U: G(u)=x\}
$$

on a neighborhood of $(G(\bar{u}), \bar{u})$.

We first recall the notion of Kuratowski's limsup:

Let $T$ be a metric space and $A_{T} \subset X \tau \in T$ be a family of subsets of $X$. The Kuratowski limsup of $A_{\tau}$ at $\tau_{0}$ is the closed set defined by

$$
\lim \sup _{\tau \rightarrow \tau_{0}} A_{\tau}=\left\{v \in X: \lim _{\tau \rightarrow \tau_{0}} \operatorname{dist}\left(v, A_{\tau}\right)=0\right\}
$$

Definition 2.1. The contingent variation of $G$ at $u \in U$ is the closed subset of $X$ defined by

$$
G^{(1)}(u):=\lim \sup _{h \rightarrow 0+} \frac{G\left(B_{h}(u)\right)-G(u)}{h}
$$

In other words $v \in G^{(1)}(u)$ is and only if

$$
\lim \inf _{h \rightarrow 0+} \operatorname{dist}\left(v, \frac{G\left(B_{h}(u)\right)-G(u)}{h}\right)=0
$$

or equivalently if there exist sequences $h_{i} \rightarrow 0+, v_{i} \rightarrow V$ such that $G(u)+h_{i} v_{i} \in G\left(B_{h_{i}}(u)\right)$. The word contingent is used because the definition reminds that of the contingent cone introduced by Bouligand (see, for example, [2]).

Clearly, $G^{(1)}(u)$ is starshaped at zero closed set, i.e. $0 \in G^{(1)}(u)$ and for all $v \in G^{1}(u), \lambda \in[0,1], \lambda v \in G^{(1)}(u)$. Let $\bar{c} o$ denote the closed convex hull and $B$ the closed unit ball in $X$.

Theorem 2.2 (Uniform Open Mapping Principle). Assume that for some $\epsilon>0, \rho>0$

$$
\rho B \subset \bigcap_{d(u, \bar{u}) \leq \varepsilon} \bar{c} o G^{(1)}(u)
$$


Then for every $u \in B_{\frac{\epsilon}{2}}(\bar{u})$ and $h \in\left[0, \frac{\epsilon}{2}\right]$ we have

$$
G(u)+h \rho \stackrel{\circ}{B} \subset G\left(B_{h}(u)\right)
$$

where $\stackrel{\circ}{B}$ denotes the open unit ball in $X$.

Proof. Fix $u, h$ as above and assume that there exists $y \in X$ satisfying

$$
|y-G(u)|<h \rho, y \quad G\left(B_{h}(u)\right)
$$

Set $\Theta^{2}=\|y-G(u)\| / h \rho$. Then $0<\Theta<1$. Applying the Ekeland variational principle $[5],[6]$ to the complete metric space $B_{h}(u)$ and the continuous function $x \rightarrow|G(x)-y|$ we prove the existence of $\bar{x} \in B_{\Theta h}(u)$ such that for all $x \in B_{h}(u)$

$$
|G(\bar{x})-y| \leq|G(x)-y|+\Theta \rho d(x, \bar{x})
$$

Observe that $\bar{x} \in \operatorname{lnt} B_{h}(u)$ and, by (2.2), $y \neq G(\bar{x})$. Hence, by differentiability of the norm, there exists $p \in X^{*}$ of $|p|=1$ such that for all $h_{j} \rightarrow 0+, v_{j} \rightarrow v$ we have

$$
\left|G(\bar{x})+h_{j} v_{j}-y\right|=|G(\bar{x})-y|+\left\langle p, h_{j} v_{j}>+o\left(h_{j}\right)\right.
$$

where $\lim _{j \rightarrow \infty} o\left(h_{j}\right) / h_{j}=0$. Fix $v \in G^{(1)}(\bar{x})$. Then from (2.3), (2.4) and Definition 2.1 we obtain

$$
0 \leq\left\langle p, h_{j} v_{j}\right\rangle+\Theta \rho h_{j}+o\left(h_{j}\right)
$$

Dividing by $h_{j}$ and taking the limit yields $\langle p, v\rangle \geq-\Theta \rho$ for all $v \in G^{(1)}(\bar{x})$. Hence

$$
\left\langle p, v>\geq-\theta \rho \text { for all } v \in \bar{c} o G^{(1)}(\bar{x})\right.
$$

Since $d(\bar{x}, \bar{u}) \leq d(\bar{x}, u)+d(u, \bar{u}) \leq \Theta h+\frac{\epsilon}{2}<\epsilon$, by $(2.1), \rho B \subset \bar{c} o G^{(1)}(\bar{x})$. This implies that

$$
-\rho \geq \inf _{v \in \bar{c} o G^{(1)}(\bar{x})}\langle p, v>\geq-\Theta \rho
$$

Since $0<\theta<1$ and $\rho>0$ we obtained a contradiction. Hence (2.2) can not hold.

Theorem 2.3. Under all assumptions of Theorem 2.2 for all $u \in B_{\frac{\epsilon}{2}}(\bar{u}), x \in B_{\frac{\epsilon}{4}}(G(u))$ we have

$$
\operatorname{dist}\left(u, G^{-1}(x)\right) \leq \frac{1}{\rho}|G(u)-x|
$$


Remark. a) Inequality (2.5) means that $G$ is pseudo-Lipschitz at $G(\bar{u}), \bar{u})$ with the Lipschitz constant $\rho^{-1}$ (see [1], [3]); b) Theorems 2.2 and 2.3 imply the main result of [3].

Theorem 2.3 follows from Theorem 2.2 and the general inverse function theorem which we prove below.

Theorem 2.4. Let $G$ be a continuous map from a complete metric space $(U, d)$ to a metric space $\left(X, d_{X}\right)$ and let $\bar{u} \in U$. Assume that form some $\rho>0, \epsilon>0,0 \leq \alpha<1$ and all $u \in B_{\epsilon}(\bar{u}), h \in[0, \epsilon]$

$$
\sup _{b \in B_{\rho h}(G(u))} \operatorname{dist}\left(b, G\left(B_{h}(u)\right)\right) \leq \alpha \rho h
$$

Then for all $h>0$ satisfying $h /(1-\alpha)+2 \rho h \leq \epsilon / 2$ and all $u \in B_{\frac{\epsilon}{2}}(\bar{u}), x \in B_{\rho h}(G(u))$ we have

$$
\operatorname{dist}\left(u, G^{-1}(x)\right) \leq \frac{1}{1-\alpha} h
$$

In particular, this implies that for all $u$ near $\bar{u}$ and all $x$ near $G(\bar{u})$

$$
\operatorname{dist}\left(u, G^{-1}(x)\right) \leq \frac{1}{\rho(1-\alpha)} d_{X}(G(u), x)
$$

Remark. When $X$ is a Banach space, assumption (2.6) can be formulated as

$$
G(u)+\rho h B \subset G\left(B_{h}(u)\right)+\alpha \rho h B
$$

and the conclusion (2.7) as

$$
\operatorname{dist}\left(u, G^{-1}(x)\right) \leq \frac{1}{\rho(1-\alpha)}|G(u)-x|
$$

Proof. Fix $h, u, x$ as above. We look for $y \in G^{-1}(u)$ as the limit of a sequence we shall built. Set $u_{0}=u$. By (2.6) there exists $u_{1}$ such that

$$
d\left(u_{0}, u_{1}\right)=d\left(u, u_{1}\right) \leq h, d_{X}\left(G\left(u_{1}\right), x\right) \leq \alpha \rho h
$$

Assume that we already constructed $u_{i}, i=1, \ldots, n$ such that

$$
d\left(u_{i-1}, u_{i}\right) \leq \alpha^{i-1} h, d_{X}\left(G\left(u_{i}\right), x\right) \leq \rho \alpha^{i} h
$$

Then 


$$
d\left(u, u_{i}\right) \leq \sum_{j=1}^{i} d\left(u_{j-1}, u_{j}\right)=h \sum_{j=0}^{i-1} \alpha^{i} \leq \frac{h}{1-\alpha}
$$

and

$$
d\left(\bar{u}, u_{i}\right) \leq d(\bar{u}, u)+d\left(u, u_{i}\right) \leq \frac{\epsilon}{2}+\frac{h}{1-\alpha} \leq \epsilon
$$

By (2.6), there exists $u_{n+1}$ such that $d\left(u_{n}, u_{n+1}\right) \leq \alpha^{n} h$ and $d_{X}\left(G\left(u_{n+1}\right), x\right) \leq \rho \alpha^{n+1} h$. Observe that (2.8) implies that $\left\{u_{i}\right\}$ is a Cauchy sequence and that $\lim _{i \rightarrow \infty} G\left(u_{i}\right)=x$. Let $y$ be the limit of $\left\{u_{i}\right\}$. Since $G$ is continuous, $G(y)=x$ and therefore $y \in G^{-1}(x)$. Moreover, by $(2.9), d(u, y) \leq h /(1-\alpha)$. .

Remark. The method applied in the proof is due to Ljusternik [13] and Graves [10].

Corollary 2.5. Let $g: X \rightarrow Y$ be a function between two Banach spaces. Assume that $g$ is continuously differentiable at some $x_{0} \in X$ and

$$
0 \in \operatorname{Int} \overline{g^{\prime}\left(x_{0}\right) B}
$$

Then for all $h>0, g\left(x_{0}\right) \in \operatorname{Int} g\left(B_{h}\left(x_{0}\right)\right)$ and there exists $L>0$ such that for all $(x, y)$ near $\left(x_{0}, g\left(x_{0}\right)\right)$

$$
\operatorname{dist}\left(x, g^{-1}(y)\right) \leq L|g(x)-y|
$$

In particular if $g\left(x_{0}\right)=0$ then for all $x \in x_{0}+\operatorname{ker}^{\prime}\left(x_{0}\right)$

$$
\operatorname{dist}\left(x, g^{-1}(0)\right)=o\left(\left\|x-x_{0}\right\|\right)
$$

and this implies that the tangent manifold to $g^{-1}(0)$ at $x_{0}$ coincides with $x_{0}+\operatorname{ker} g^{\prime}\left(x_{0}\right)$.

Remark. We observe that the assumption $(2.10)$ is verified whenever $g^{\prime}\left(x_{0}\right)$ is surjective, i.e.

$$
g^{\prime}\left(x_{0}\right) X=Y
$$

Indeed $g^{\prime}\left(x_{0}\right) X=\bigcup_{n \geq 1} n g^{\prime}\left(x_{0}\right) B$ and, by Baire's theorem, for some $n \geq 1$ the set $n g^{\prime}\left(x_{0}\right) B$ has a nonempty interior. Hence Int $g^{\prime}\left(x_{0}\right) B \neq \varnothing$ and, using that $g^{\prime}\left(x_{0}\right) B=-g^{\prime}\left(x_{0}\right) B$ is a convex set we obtain

$$
0 \in \operatorname{Int} g^{\prime}\left(x_{0}\right) B \subset \operatorname{Int} \overline{g^{\prime}\left(x_{0}\right) B}
$$

Thus Corollary 2.5 extends Ljusternik's theorem [13]. 


\section{Interior Points of Reachable Sets}

Let $U$ be a topological space, $X$ be a reflexive Banach space with the norm Gateaux differentiable away from zero and $f: X \times U \rightarrow X$ be a continuous differentiable in the first variable function. We assume that

a) $f$ is locally Lipschitz in the first variable uniformly on $U$, i.e. for all $x \in X$ there exist $L>0$ and $\epsilon>0$ such that for all $u \in U, f(\cdot, u)$ is $L$-Lipschitz on $B_{\epsilon}(x)$ :

$$
\left\|f\left(x^{\prime}, u\right)-f\left(x^{\prime \prime}, u\right)\right\| \leq L\left\|x^{\prime}-x^{\prime \prime}\right\|, \text { for all } x^{\prime}, x^{\prime \prime} \in B_{\epsilon}(x)
$$

b) For all $u \in U$ the derivative $\frac{\partial f}{\partial x}(\cdot, u)$ is continuous

c) For all $x \in X$ the set $f(x, U)$ is bounded

For all $T>0$ a (Lebesgue) measurable function $u:[0, T] \rightarrow U$ is called an admissible control. Let $Q_{T}$ denote the set of all admissible controls defined on the time interval $[0, T]$. Define a metric on $Q_{T}$ by setting

$$
d_{T}(u, v)=\mu(\{t \in[0, t]: u(t) \neq v(t)\})
$$

where $\mu$ denotes the Lebesgue measure. Then the space $\left(Q_{T}, d_{T}\right)$ is complete (see Ekeland [6]).

Let $\{S(t)\}_{t \geq 0}$ be a strongly continuous semigroup of linear operators from $X$ to $X$ and $A$ be its infinitesimal generator, $x_{0} \in X$. Consider the control system

$$
\left\{\begin{array}{l}
x^{\prime}(t)=A x(t)+f(x(t), u(t)), u \in Q_{T}, T>0 \\
x(0)=x_{0}
\end{array}\right.
$$

Recall that a continuous function $x:[0, T] \rightarrow X$ is called a mild trajectory of (3.1) if for some $u \in Q_{T}$ and all $0 \leq t \leq T$

$$
x(t)=S(t) x_{0}+\int_{0}^{t} S(t-s) f(x(s), u(s)) d s
$$

We denote by $x_{u}$ the trajectory corresponding to the control $u$. Define the reachable set of (3.1) at time $T>0$ by

$$
R(T)=\{x(T): x \in C(0, T ; X) \text { is a mild trajectory of }(3.1)\} .
$$

Let $z$ be a mild trajectory of (3.1) on $[0, T]$ and $\bar{u}$ be the corresponding control. In this section we provide a sufficient condition for

$$
z(T) \in \operatorname{Int} R(T)
$$


and for the regularity of the "inverse." Consider the linear control system

$$
\begin{cases}w^{\prime}(t)= & A w(t)+\frac{\partial f}{\partial x}(z(t), \bar{u}(t)) w(t)+v(t) \\ w(0)= & 0 \\ v(t) \in & \bar{c} o f(z(t), U)-f(z(t), \bar{u}(t))\end{cases}
$$

and let $R^{L}(T)$ denote the corresponding reachable set of $(3.3)$ at time $T$. Let $S_{\bar{u}}(t ; s)$ denote the solution operator of the equation

$$
Z^{\prime}(t)=A Z(t)+\frac{\partial f}{\partial x}(z(t), \bar{u}(t)) Z(t)
$$

where $X_{\bar{u}}(s ; s)=I d$. Then

$$
R^{L}(T)=\left\{\int_{0}^{T} S_{\bar{u}}(T ; s) v(s) d s: v(s) \in \bar{c} o f(z(s), U)-f(z(s), \bar{u}(s))\right\}
$$

Theorem 3.1. Assume that $0 \in \operatorname{lnt} R^{L}(T)$. Then $z(T) \in \operatorname{Int} R(T)$ and there exist $\epsilon>0, L>0$ such that for every control $u \in Q_{T}$ satisfying $d_{T}(u, \bar{u}) \leq \epsilon$ and all $b \in B_{\epsilon}(z(T))$ there exists a trajectory-control pair $\left(x_{\hat{u}}, \hat{u}\right)$ which verifies

$$
x_{\hat{u}}(T)=b ; d_{T}(u, \hat{u})<L\left\|b-x_{u}(T)\right\|
$$

In particular for all $b \in B_{\epsilon}(z(T))$ there exists a control $u \in Q_{T}$ such that

$$
\mu(\{t \in[0, T]: u(t) \neq \bar{u}(t)\}) \leq L|b-z(T)|
$$

and the trajectory $x_{u}$ corresponding to this control verifies $x_{u}(T)=b$.

Proof. Replacing $t$ by $t / T$ we may assume that $T=1$. Set $Q=Q_{1}, d=d_{1}$. For all $u \in Q$, let $x_{u}$ be the solution of $(3.1)$ (when it exists on $[0,1]$ ) corresponding to the control $u$.

From the Gronwall inequality follows that for some $\delta>0$ the map $\varphi(u)=x_{u}$ from $B_{2 \delta}(\bar{u})$ to $C(0,1 ; E)$ is well defined and is Lipschitzian. For all $u \in B_{\delta}(\bar{u})$ and $s \in[0,1]$, let $S_{u}(\cdot ; s)$ denote the solution operator of the equation

$$
\left.Z^{\prime}(t)=A Z(t)+\frac{\partial f}{\partial x}\left(x_{u}(t)\right), u(t)\right) Z(t)
$$

Fix $u \in B_{\delta}(\bar{u})$ and $v \in U$. For all $\left.t_{0} \in\right] 0,1[, h>0$ we consider the needle perturbations of controls 


$$
u_{h}(t)= \begin{cases}v & t_{0}-h<t \leq t_{0} \\ u(t) & \text { otherwise }\end{cases}
$$

Let $x_{h}$ denote the solution of (3.1) corresponding to the control $u_{h}$. It is well known that at every Lebesgue point $t_{0}$ of the function $f\left(x_{u}(\cdot), u(\cdot)\right)$ we have

$$
\lim _{h \rightarrow 0+} \frac{x_{h}(1)-x_{u}(1)}{h}=S_{u}\left(1 ; t_{0}\right)\left(f\left(x_{u}\left(t_{0}\right), v\right)-f\left(x\left(t_{0}\right), u\left(t_{0}\right)\right)\right)
$$

(see for example Fattorini [7]). Set $V_{u}(t)=f\left(x_{u}(t), U\right)-f\left(x_{u}(t), u(t)\right.$ ) and define the continuous map $G: B_{2 \delta}(\bar{u}) \rightarrow X$ by

$$
G(u)=x_{u}(1)
$$

Then, by (3.4), for all $u \in B_{\delta}(\bar{u})$, and for almost all $t_{0} \in[0,1]$ and all $v \in V_{u}\left(t_{0}\right)$ $S_{u}\left(1 ; t_{0}\right) v \in G^{(1)}(u)$. Therefore for all $v \in \bar{c} o V_{u}\left(t_{0}\right), S_{u}\left(t ; t_{0}\right) v \in \bar{c} o G^{(1)}(u)$. Hence, by the mean value theorem, for all measurable selection $v(t) \in \bar{c} o V_{u}(t)$

$$
\int_{0}^{1} S_{u}(1 ; t) v(t) d t \in \overline{c o} G^{(1)}(u)
$$

Let $\rho>0$ be such that

$$
\rho B \subset\left\{\int_{0}^{1} S_{\bar{u}}(1 ; t) v(t) d t: v(t) \in \overline{c o} V_{\bar{u}}(t)\right\}
$$

The Gronwall inequality implies that $S_{u}(1 ; \cdot) \rightarrow S_{\bar{u}}(1 ; \cdot)$ uniformly when $u \rightarrow \bar{u}$ and

$$
\lim _{u \rightarrow \bar{u}} \int_{0}^{1} H\left(\overline{c o} V_{\bar{u}}(t), \overline{c o} V_{u}(t)\right) d t=0
$$

where $H$ states for the Hausdorff distance. Since the right-hand side of (3.5) is convex and closed this yields that for some $\delta^{\prime}>0$ and all $u \in B_{\delta^{\prime}}(\bar{u})$

$$
\frac{\rho}{2} B \subset\left\{\int_{0}^{1} S_{u}(1 ; t) y(t) d t: y(t) \in \overline{c o} V_{\bar{u}}(t)\right\} \subset \overline{c o} G^{(1)}(u)
$$

Theorem 2.3 ends the proof.

Remark. Recall that in infinite dimensions the linear system

$$
x^{\prime}=A x+B u, \quad u \in U
$$

where $U$ is a Banach space and $B \in L(U, X)$ is not in general exactly controllable by 
$L^{p}(0, T ; U)$ controls $p>1$ ) (see R. Triggiani [20], [21], J.C. Louis and D. Wexler [14]). Therefore, when $U$ is a bounded subset of a Banach space we can neither expect (in general) the reachable sets of (3.3) to have a nonempty interior. The results from $[4],[17]$ give an idea of what has to be assumed about the semigroup $S$ and the operator $B$ to get the exact local reachability of (3.3) at zero. They also indicate how narrow the class of such systems is. In the next section we apply Theorem 3.1 to a nonlinear problem of local exact reachability. 


\section{A Local Reachability Problem}

Let $X$ be a reflexive, $E$ be a separable reflexive Banach space, $A$ be the infinitesimal generator of a $C_{0}$-semigroup $S(t) \in L(X, X), t \geq 0, B \in L(E, X)$. Consider a topological space $U$ and a continuous function $f: X \times U \rightarrow E$. We assume that $f$ satisfies all the assumptions from Section 3 . We study here the control system

$$
\left\{\begin{array}{l}
x^{\prime}=A x+B f(x, u), u \in U \\
x(0)=0
\end{array}\right.
$$

Theorem 4.1. Assume that form $\bar{u} \in U, f(0, \bar{u})=0, \frac{\partial f}{\partial x}(0, \bar{u})=0$ and that

$$
\inf _{p \in X^{*},|p|=1} \int_{0}^{T} \sup _{u \in U}<B^{*} S(s)^{*} p, f(0, u)>d s>0
$$

where $T>0$ is a given time. Then for some $L>0$ and all $x_{0}$ near zero there exists a measurable control $u(s) \in U$ such that the corresponding trajectory $x_{u}$ satisfies

$$
x_{u}(T)=x_{0} \text { and } \mu(\{t: u(t) \neq 0\}) \leq L\left\|x_{0}\right\|
$$

Remark. Observe that for all $p \in X^{*}$

$$
\sup _{u \in U}<B^{*} S(s)^{*} p, f(0, u)>=\sup _{v \in \bar{c} o f(0, U)}<B^{*} S(s)^{*} p, v>
$$

Therefore, from $[17$, Proposition 2.2] we deduce that the function $s \rightarrow \sup _{u \in U}<B^{*} S(s)^{*} p, f(0, u)>$ is integrable. Hence the integral in (4.2) is well defined.

Some corollaries are in order.

Corollary 4.2. Assume that for some $\bar{u} \in U, f(0, \bar{u})=0, \frac{\partial f}{\partial x}(0, \bar{u})=0$ and $0 \in \operatorname{Int} \overline{c o} f(0, U)$. If

$$
\inf _{p \in X^{*},|p|=1}\left|B^{*} S(\cdot)^{*} p\right|_{L^{1}\left(0, T ; E^{*}\right)}>0
$$

then the conclusion of Theorem 4.1 is valid.

Corollary 4.3. Under the assumptions of Corollary 4.2 assume that $B$ is surjective and that for some $t_{0}>0, S\left(t_{0}\right)$ is surjective. Then the conclusion of Theorem 4.1 is valid.

Corollary 4.4. In Theorem 4.1 assume that $U$ is a bounded subset of a separable reflexive Banach space $E$ and $f(x, u)=g(x)+u$, where $g$ is $C^{1}$ on a neighborhood of zero. If $g(0)=0, g^{\prime}(0)=0,0 \in U$ and 


$$
\inf _{p \in X^{*},|p|=1} \int_{0}^{T} \sup _{u \in U}<B^{*} S(s)^{*} p, u>d s>0
$$

then the conclusion of Theorem 5.1 is valid.

Proof of Theorem 4.1. By Theorem 3.1 we have to show that $0 \in \ln R^{L}(T)$, where $R^{L}(T)$ denotes the reachable set at time $T$ of the linear system.

$$
x^{\prime}=A x+v, v \in \overline{c o} B f(0, U)
$$

The set $\overline{c o} f(0, U)$ being weakly sequentially compact, we know that $B \overline{c o} f(0, U)$ is a closed convex set. Hence $\overline{c o} B f(0, U) \subset B \overline{c o} f(0, U)$. Moreover $B \operatorname{cof}(0, U) \subset \overline{c o} B f(0, U)$. Therefore we proved that $\bar{c} o B f(0, U)=B \bar{c} o f(0, U)$. Thus the system (4.4) may be replaced by the linear control system

$$
\left\{\begin{array}{l}
x^{\prime}=A x+B v, v \in \overline{c o} f(0, U) \\
x(0)=0
\end{array}\right.
$$

The admissible controls $U_{a d}(T)$ are measurable selections of $\bar{c} o f(0, u)$ defined on the time interval $[0, T]$. By [17] the reachable set $R^{L}(T)$ of $(4.5)$ at tome $T$ is weakly compact. Clearly $R^{L}(T)$ is convex. Thus, by the separation theorem, we shall end the proof when we show that

$$
\inf _{p \in X^{*}, \mathrm{l} p \mathrm{l}=1} \sup \left\{\left\langle p, x>: x \in R^{L}(T)\right\}>0\right.
$$

By [15], for all $p \in X$

$$
\begin{aligned}
\sup & \left\{<p, x>: x \in R^{L}(T)\right\}=\sup \left\{\int_{0}^{T}<p, S(T-s) B u(s)>d s: u \in U_{a d}(T)\right\} \\
& =\int_{0}^{T} \sup _{u \in \operatorname{cof}(0, U)}<B^{*} S(T-s)^{*} p, u>d s=\int_{0}^{T} \sup _{u \in U}<B^{*} S(T-s) p, f(0, u)>d s
\end{aligned}
$$

and therefore (4.6) follows from the assumption (4.2).

Proof of Corollary 4.2. Let $\gamma>0$ be such that $\left\{v \in E:\|v\|_{E} \leq \gamma\right\} \subset \bar{c} \circ f(0, U)$. Then $\sup _{u \in U}<B^{*} S(s)^{*} p, f(0, u)>\geq \gamma B^{*} S(s)^{*} p \mid$ and therefore (4.3) implies (4.2). Theorem 4.1. ends the proof.

Proof of Corollary 4.3. Since $S\left(t_{0}\right)$ is surjective, by [14], $S(t)$ is surjective for all $t \geq 0$ and therefore $S(t) B$ is surjective. Let $\gamma>0$ be as in the proof of Corollary 4.2. By a Banach theorem, for every $t \geq 0$ there exists $\rho(t)>0$ such that

$$
\{x \in X:\|x\| \leq \rho(t)\} \subset S(t) B\left(\left\{v \in E:\|v\|_{E} \leq \gamma\right\}\right)
$$


Indeed, by Corollary 4.2 and the assumption (4.9) we have to show that

$$
\inf _{p \in X^{*},|p|=1} \int_{0}^{T} \mid B^{*} S(t)^{*} p \|_{L^{2}(0,1)} d t>0
$$

By $\left[4\right.$, p. 58] there exists $\gamma>0$ such that for all $p \in X^{*}$

$$
\left\|B^{*} S(\cdot)^{*} p\right\|_{L^{2}\left(0, T ; L^{2}(0,1)\right)} \geq \gamma\|p\|
$$

On the other hand for some $M>0$ and all $p \in X^{*}$ of $|p|=1$

$$
\int_{0}^{T}\left\|B^{*} S(t)^{*} p\right\|_{L^{2}(0,1)} d t \geq \underset{0}{T}\left\|B^{*} S(t)^{*} p\right\|^{2}{ }_{L^{2}(0,1)} d t=M\left\|B^{*} S(\cdot)^{*} p\right\|_{L^{2}\left(0,1 ; L^{2}(0,1)\right)}^{2} \geq M \gamma^{2}
$$

This implies (4.10) and ends the proof of our claim. 


\section{References}

[1] Aubin J.P. [1982] Comportement Lipschitzien des solutions de problemes de minimisation convexes. CRAS 295, 235-238.

[2] Aubin J.P. and I. Ekeland [1984] Applied Nonlinear Analysis. Wiley Interscience, New York.

[3] Aubin J.P. and H. Frankowska [1087] On inverse function theorems for set-valued maps. J. Math Pure Appl. 66, pp. 71-89.

[4] Curtain R.F., A.J. Pritchard [1978] Infinite Dimensional Linear Systems Theory, Lecture Notes in Control and Information Sciences, Springer Verlag.

[5] Ekeland 1. [1974] On the variational principle, J. Math. Anal. Appl. 47 pp. 324-358.

[6] Ekeland I. [1979] Nonconvex minimization problems, Bull. Am. Math. Soc. 1, pp. 443-474.

[7] Fattorini M. [1987] A unified theory of necessary conditions for nonlinear nonconvex control systems, Applied Mathematics and Optimization, Vol. 2, pp. 141-184.

[8] Frankowska H. An open mapping principle for set-valued maps, J. of Math. Analysis and Appl. (to appear).

[9] Frankowska H. Local controllability of control systems with feedback. J. of Optimization Theory and Applications (to appear).

[10] Graves L.M. [1950] Some mapping theorem, Duke Math. J. 17, pp. 111-114.

[11] Lions J.L. [1971] Optimal Control of Systems Described by Partial Differential Equations, Springer.

[12] Lions J.L. and E. Magenes [1968-1970] Problèmes aux limites non homogènes, 3 Vols, Dunod, Paris.

[13] Ljusternik L.A. [1934] Conditional extrema of functionals. Mat. Sb. 41, pp. 390-401.

[14] Louis J.L. and D. Wexler, On exact controllability in Hilbert spaces, in Trends in Theory and Practice of Nonlinear Differential equations, ed. Lakshmikantham, Lecture Notes in Pure and applied Mathematics, Vol. 90, Marcel Dekker INC, Bew York and Basel.

[15] Magnusson K., Pritchard A.J. and M.D. Quinn [1985] The application of fixed point theorems to global nonlinear controllability problems, in Mathematical control theory, Banach Center Publications, Vol. 14, pp. 319-343.

[16] Magnusson K. and A.J. Pritchard [1981] Local Exact Controllability of Nonlinear Evolution Equations, in Recent Advances in Differential Equations, Academic Press, pp. 271-280. 
[17] Peichl G. and W. Schappacher [1986] Constrained Controllability in Banach Spaces, SIAM J. on Control and Optimization, (24), pp. 1261-1275.

[18] Quinn M.D. and N. Carmichael [1984-85] An approach to non-linear control problems using fixed point methods, degree theory and pseudo-inverses, Numer. Funct. Anal. and Optimiz. 7(283) pp. 197-219.

[19] Russel D. [1978] Controllability and stability theory for linear partial differential equations: recent progress and open questions, SIAM Review, pp. 639-739.

[20] Triggiani R. [1975] Controllability and observability in Banach space with bounded operators, SIAM J. on Control and Optimization, (13), pp. 462-491.

[21] Triggiani R. [1980] A note on the lack of exact controllability for mild solutions in Banach spaces, SIAM J. on Control and Optimization, (18) , pp. 98-99.

[22] Yorke J. [1972] The maximum principle and controllability of nonlinear differential equations, SIAM J. of Control and Optimization, (10) pp. 334-338. 